\title{
Nanostructured Fishnet Silicon Photodetector Pixels as a Fully-Contained Microspectrometer Chip
}

\author{
Jasper J. Cadusch*1, Jiajun Meng ${ }^{1}$ and Kenneth B. Crozier*1,2 \\ ${ }^{1}$ Department of Electrical and Electronic Engineering, University of Melbourne, Victoria 3010, Australia \\ ${ }^{2}$ School of Physics, University of Melbourne, Victoria 3010, Australia \\ *-Emails: caduschj@unimelb.edu.au, kenneth.crozier@unimelb.edu.au
}

\begin{abstract}
We experimentally demonstrate a microspectrometer comprising twenty silicon photodetector pixels, whose responsivities are engineered via nanostructured fishnet patterns. We computationally reconstruct the spectrum of light that illuminates the chip from the measured pixel photocurrents.
\end{abstract}

OCIS codes: 350.4238, 040.5160, 120.6200 .

\section{Introduction}

Conventional spectrometers generally combine broadband photodetectors with a device for spectral selection such as a grating or a Michelson interferometer. For applications in which minimized size and weight are important, filter arrays provide an alternative means for spectral selection. Bao and Bawendi, for example, demonstrated a filter array based on quantum dots spotted by pipette onto a glass coverslip [1]. This filter array was attached to a monochrome image sensor, thereby achieving a compact spectrometer. Here we present a device where the spectral filtering and photon detection occur within the same silicon structure. The all-silicon nature of our device has advantages from a manufacturing standpoint. We experimentally demonstrate that nanostructuring with a fishnet pattern allows the responsivity spectra of silicon photodiodes to be engineered. We demonstrate that the spectrum of light illuminating the chip can be reconstructed by an algorithm that takes as inputs the photocurrents measured from twenty fishnet pixels and the measured responsivity spectra. This work thus achieves a very small footprint all-silicon spectrometer.
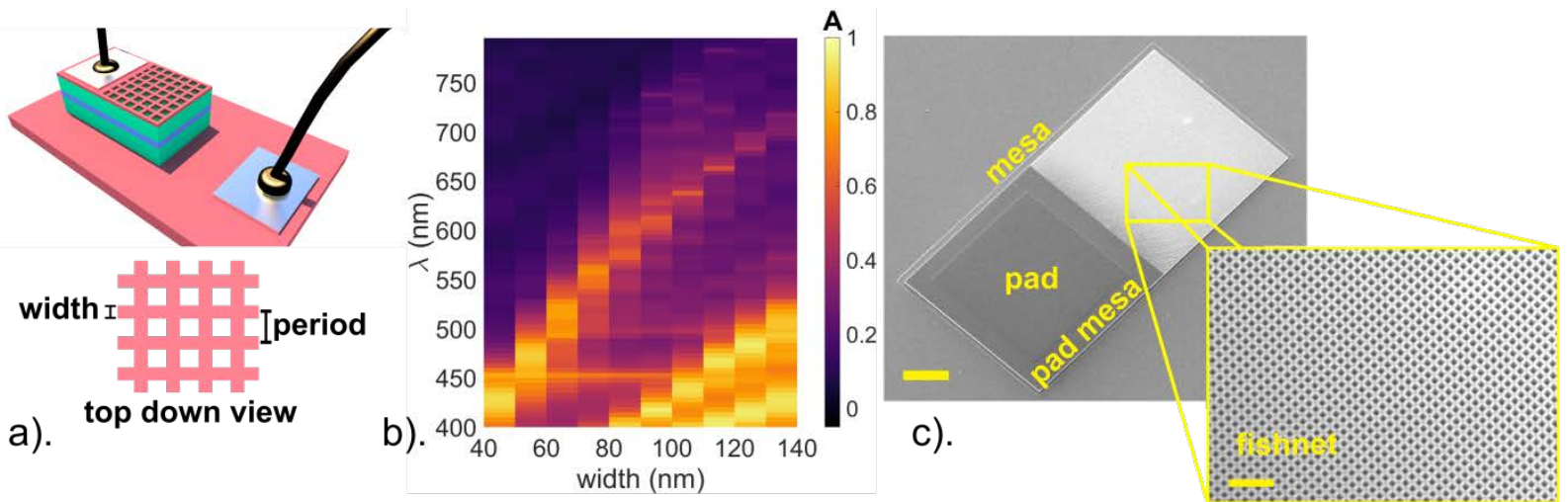

Fig. 1: a). Schematic of fishnet pixel. $p^{+}, n^{-}$and $n^{+-d o p e d ~ r e g i o n s ~ a r e ~ s h o w n ~ i n ~ r e d, ~ g r e e n ~ a n d ~ b l u e ~ r e s p e c t i v e l y . ~ b) . ~ S i m u l a t e d ~ f r a c t i o n a l ~}$ absorption vs wavelength and fish-net width. c). SEM of fishnet pixel. Scale bar: $50 \mu \mathrm{m}$. Inset: zoom-in of fishnet structure. Scale bar: $2 \mu \mathrm{m}$.

A single fishnet pixel is illustrated as Fig. 1a. The red, green and blue layers represent $p^{+}, n^{-}$and $n+$-doped silicon regions respectively. The $n+$ (blue) layer is the bottom of the etched fish-net region, below which is a solid Si mesa, similar to the design in [2]. Each pixel thus contains two back-to-back P-I-N diodes and can thus provide two photocurrent measurements, one for each bias polarity. In other words, when the top contact is biased negatively with respect to the bottom contact, the upper and lower diodes are reverse- and forward-biased, respectively. The measured photocurrent thus originates from light absorbed in the top (fishnet) diode. Similarly, when the bias polarity is changed, the lower (mesa) diode is reverse biased, so that the measured photocurrent originates from light absorbed there, with the fishnet region acting as an absorptive optical filter. The fishnet absorption can be understood by thinking of it as comprising orthogonally-oriented slab waveguides. The widths of these waveguides control the dispersion of optical modes they support. Finite element method (FEM) simulations of the fraction of 
(normally-incident) light absorbed in the lightly-doped ( $n$-) region of the fishnet vs wavelength and fishnet width, for a fixed array period of $500 \mathrm{~nm}$, is shown as Fig. 1b. These simulations clearly demonstrate that the wavelength(s) of peak absorption can be tuned by changing the waveguide width. We demonstrate that we can employ this to engineer the responsivities of our pixels.

\section{Results and Discussion}

The starting substrate for our device is $p^{+}$doped, on which $p^{+}, n-, n+$, and $n$ - layers with thicknesses of $0.2 \mu$ m, 2 $\mu \mathrm{m}, 1 \mu \mathrm{m}$ and $4 \mu \mathrm{m}$ respectively, are formed. The fishnet is defined by e-beam lithography to define an aluminum (Al) etch mask $(60 \mathrm{~nm})$ for a subsequent reactive ion etching step. This results in the fishnets being $\sim 2.5 \mu \mathrm{m}$ deep. The mesa is then produced by photolithography and reactive ion etching (to depth of $\sim 6 \mu \mathrm{m}$ ). The device is completed by adding $\mathrm{Al}$ contacts by photolithography and evaporation, with the result shown as Fig. 1c.
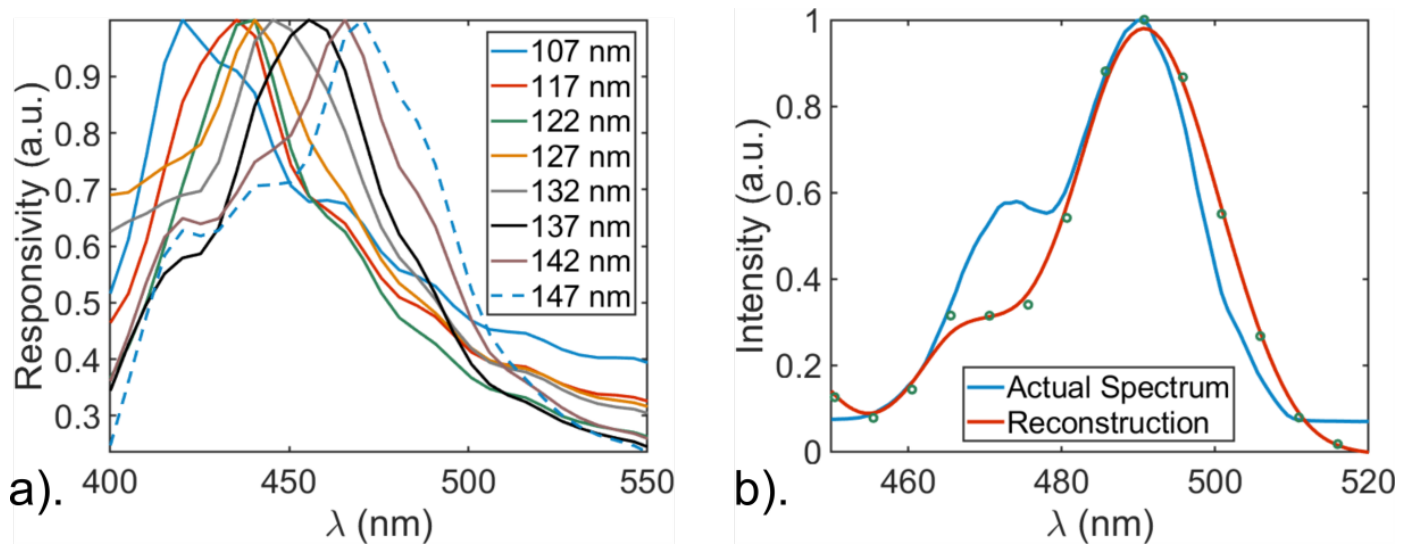

Fig. 2: a). Normalized responsivity spectra of eight fishnet photodetectors, for which the fishnets are of different widths. b). Spectrum of light from supercontinuum source (passed through two filters and a dichroic), as measured by our device (green circles and red line spline fit) and by reference spectrometer (Ocean Optics QE-Pro, blue line)

We measure the responsivities of our pixels by illuminating them with light of known power and wavelength. This is provided by a laser driven white light source (Energetiq EQ-99X) coupled to a monochromator (Princeton Instr Acton SP2150). The photocurrent from each pixel vs wavelength for voltages of +1.5 and $-1.5 \mathrm{~V}$ are recorded by a picoammeter (Keithley 2502). The normalized results (Fig. 2a) show an appreciable shift in peak responsivity wavelength with increasing fish-net waveguide width. We reconstruct incident spectra by treating the measured photocurrents and measured responsivities as inputs to a linear least-squares problem, employing Tikhonov regularization for solution stability [3]. We illuminate our device with light from a supercontinuum source (NKT SuperK Compact) that is passed through two shortpass filters and a dichroic mirror. We reconstruct the spectrum of this light using the photocurrents from 20 pixels (i.e. 40 photocurrent measurements). The results (Fig. 2b) are in good agreement with the spectrum measured by a reference spectrometer (Ocean Optics QE-Pro). This is especially true for the wavelength and linewidth of the main peak, while the secondary peak is only partially resolved. It should be noted that the wavelength-dependence of the reference system (QE-Pro) has not been accounted for.

\section{Conclusion}

We have demonstrated a CMOS-compatible nanophotonic device that functions as a fully-contained spectrometer. The fish-net can be tuned to absorb different wavelengths, enabling engineering of the responsivity spectrum. Our approach could find use in portable consumer electronics for color-matching, sample analysis or multi-spectral imaging.

\section{References}

[1] J. Bao and M. G. Bawendi, “A colloidal quantum dot spectrometer,” Nature 523, 67-70 (2015).

[2] H. Park and K. B. Crozier, "Vertically stacked photodetector devices containing silicon nanowires with engineered absorption spectra," ACS Photonics 2, 544-549 (2015).

[3] U. Kurokawa, B. I. Choi, and C.-C. Chang, "Filter-based miniature spectrometers: spectrum reconstruction using adaptive regularization,” IEEE Sensors Journal 11, 1556-1563 (2011). 


\section{University Library}

\section{- M M N E R VA A gateway to Melbourne's research publications}

Minerva Access is the Institutional Repository of The University of Melbourne

Author/s:

Cadusch, JJ;Meng, J;Crozier, KB

Title:

Nanostructured Fishnet Silicon Photodetector Pixels as a Fully-Contained Microspectrometer Chip

Date:

2018-01-01

Citation:

Cadusch, J. J., Meng, J. \& Crozier, K. B. (2018). Nanostructured Fishnet Silicon Photodetector Pixels as a Fully-Contained Microspectrometer Chip. 2018 CONFERENCE ON LASERS AND ELECTRO-OPTICS (CLEO), Part F94-CLEO_SI 2018, IEEE. https:// doi.org/10.1364/CLEO_SI.2018.SM2I.4.

Persistent Link:

http://hdl.handle.net/11343/294889 